A.B. Kharazishvili, A. Razmadze Mathematical Institute, M. Alexidze Street 1, Tbilisi 0193, Republic of Georgia. email: kharaz2@yahoo.com

\title{
A NONSEPARABLE EXTENSION OF THE LEBESGUE MEASURE WITHOUT NEW NULLSETS
}

\begin{abstract}
Under the Continuum Hypothesis, it is shown that there exists a nonseparable extension of the Lebesgue measure on the real line whose nullsets coincide with the nullsets in the Lebesgue sense.
\end{abstract}

Let $E$ be a set, $\mathcal{S}$ be a $\sigma$-algebra of subsets of $E$ containing all one-element subsets (singletons) of $E$, and let $\mu$ be a nonzero $\sigma$-finite continuous (i.e., vanishing at all singletons) measure on $\mathcal{S}$. The general measure extension problem is to extend $\mu$ to a maximally large class of subsets of $E$. According to Ulam's theorem (see, for instance, [8] or [5], Chapter 5), it is consistent with the axioms of set theory that the domain of any extension $\mu^{\prime}$ of $\mu$ cannot coincide with the power set of $E$ (for instance, this is so if $\operatorname{card}(E)$ is smaller than the first inaccessible cardinal number). Consequently, there always exists a set $X \subset E$ such that $X \notin \operatorname{dom}\left(\mu^{\prime}\right)$. An easy argument shows that $\mu^{\prime}$ can be extended to a measure $\mu^{\prime \prime}$ so that $X$ becomes $\mu^{\prime \prime}$-measurable (see, e.g., Example 2 below). Thus, assuming that there are no large cardinals, one can infer that there are no maximal extensions of the original measure $\mu$.

In this context, a similar result should be mentioned, which states that if $\left\{X_{i}: i \in I\right\}$ is an arbitrary partition of $E$, then there exists a measure $\nu$ on $E$ extending $\mu$ and satisfying the relation

$$
\left\{X_{i}: i \in I\right\} \subset \operatorname{dom}(\nu) .
$$

For the proof of this result, see [2] or Cor. 2 in [1], p. 3. It should be noticed that measures in [1] and [2] are assumed to be probability ones, but the same argument works for arbitrary $\sigma$-finite measures. Actually, we do not need the

Key Words: Continuum Hypothesis, measure extension problem, Lebesgue measure, nullset, nonseparable extension of measure

Mathematical Reviews subject classification: Primary 28A05; Secondary 28D05

Received by the editors February 7,2007

Communicated by: Krzysztof Chris Ciesielski 
above-mentioned result in our further consideration, but it also shows that, having any finite family of subsets of $E$, one can always extend $\mu$ to a measure which measures all these subsets (it suffices to consider the finite partition of $E$ generated by the given family).

An analogous assertion fails to be true for arbitrary countable families of subsets of $E$. Moreover, the existence of a Luzin set $L$ on the real line $\mathbb{R}$ having the cardinality of the continuum (see, e.g., [5], Chapter 20) implies that there is a countably generated $\sigma$-algebra of subsets of $\mathbb{R}$ containing all singletons and not admitting any nonzero $\sigma$-finite continuous measure. Indeed, it can easily be shown that every $\sigma$-finite continuous Borel measure given on a separable metric space is concentrated on some first category subset of that space. Since all first category subsets of $L$ are at most countable, it follows that $L$ does not admit a nonzero $\sigma$-finite continuous measure on the countably generated Borel $\sigma$-algebra of $L$. Now, using a one-to-one correspondence between $L$ and $\mathbb{R}$, we obtain the required $\sigma$-algebra of subsets of $\mathbb{R}$. In a certain sense, a Luzin set $L$ is a small subset of $\mathbb{R}$ because it has outer measure zero with respect to any Borel $\sigma$-finite continuous measure on $\mathbb{R}$. Recall that the existence of Luzin sets needs some additional set-theoretical axioms. For instance, the Continuum Hypothesis readily implies that there are Luzin subsets of $\mathbb{R}$ (see [5], Chapter 20). Similarly to this, Martin's Axiom implies that there are so-called generalized Luzin subsets of $\mathbb{R}$. In this connection, it should be underlined that the existence of analogous small subsets of $\mathbb{R}$ having cardinality $\omega_{1}$ can be established within the theory ZFC (see, e.g., [6] or [9]).

All measures considered below are assumed to be nonzero, $\sigma$-finite, and continuous. If the need arises, we can additionally suppose, without loss of generality, that a measure under consideration is also complete (replacing it by its completion).

For any complete measure $\mu$, we denote by $\mathcal{I}(\mu)$ the $\sigma$-ideal of all $\mu$-measure zero sets (in short, $\mu$-nullsets).

The symbol $\mu^{*}$ (respectively, $\mu_{*}$ ) denotes the outer (respectively, inner) measure associated with $\mu$.

The symbol $\lambda$ stands for the Lebesgue measure on $\mathbb{R}$ (recall that $\lambda$ is a complete measure).

The symbols $\omega$ and $\mathbf{c}$ denote, respectively, the least infinite cardinal and the cardinality of the continuum.

Example 1. A well-known method of extending a given complete measure $\mu$ is based on adding to $\mathcal{I}(\mu)$ some new sets, which are nonmeasurable with respect to $\mu$ and whose inner $\mu$-measure is equal to zero (cf. [7]). Proceeding in this way, we come to a $\sigma$-ideal $\mathcal{I}^{\prime}$ which properly contains $\mathcal{I}(\mu)$ and all of whose elements are of inner $\mu$-measure zero. Then we consider the $\sigma$-algebra $\mathcal{S}^{\prime}$ generated by $\operatorname{dom}(\mu)$ and $\mathcal{I}^{\prime}$. Any element $U$ of this $\sigma$-algebra admits a 
representation $U=(X \cup Y) \backslash Z$ where $X \in \operatorname{dom}(\mu), Y \in \mathcal{I}^{\prime}$ and $Z \in \mathcal{I}^{\prime}$. We define a functional $\mu^{\prime}$ on $\mathcal{S}^{\prime}$ by the formula

$$
\mu^{\prime}(U)=\mu^{\prime}((X \cup Y) \backslash Z)=\mu(X) .
$$

A straightforward verification shows that functional $\mu^{\prime}$ is well defined and that $\mu^{\prime}$ is also a measure on $\mathcal{S}^{\prime}$ extending the initial measure $\mu$.

Example 2. The method of extending measures described in Example 1 has a weak side. Indeed, from the viewpoint of the theory of Boolean algebras, the complete measure $\mu$ and its extension $\mu^{\prime}$ are the same. Nevertheless, slightly changing the above method, we can achieve some difference between a measure and its extension if both of them are considered on the corresponding quotient Boolean algebras. For this purpose, let us take any set $T \subset E$ nonmeasurable with respect to a complete measure $\mu$. Obviously, we must have $\mu_{*}(T)<\mu^{*}(T)$. If $T_{0}$ denotes a $\mu$-measurable kernel of $T$ and $T_{1}$ stands for a $\mu$-measurable hull of $T$, then $\mu\left(T_{1} \backslash T_{0}\right)>0$, and the set $T \backslash T_{0}$ being a subset of $T_{1} \backslash T_{0}$ satisfies the equalities

$$
\mu_{*}\left(T \backslash T_{0}\right)=\mu_{*}\left(\left(T_{1} \backslash T_{0}\right) \backslash\left(T \backslash T_{0}\right)\right)=0 .
$$

So we may assume (replacing, if necessary, $T$ by $T \backslash T_{0}$ and $E$ by $T_{1} \backslash T_{0}$ ) that

$$
\mu_{*}(T)=\mu_{*}(E \backslash T)=0 .
$$

Let $\mathcal{S}^{\prime}$ denote the $\sigma$-algebra of all those subsets $U$ of $E$, which admit a representation

$$
U=(X \cap T) \cup(Y \cap(E \backslash T)),
$$

where $X \in \operatorname{dom}(\mu)$ and $Y \in \operatorname{dom}(\mu)$. Define a functional $\mu^{\prime}$ on $\mathcal{S}^{\prime}$ by the formula

$$
\mu^{\prime}(U)=(1 / 2)(\mu(X)+\mu(Y)) .
$$

As earlier, $\mu^{\prime}$ is well defined and turns out to be a measure on $\mathcal{S}^{\prime}$ extending $\mu$. Also, since $T$ is $\mu^{\prime}$-measurable, $\mu^{\prime}$ strictly extends $\mu$. Moreover, we see that the quotient Boolean algebra associated with $\mu$ is properly contained in the quotient Boolean algebra associated with $\mu^{\prime}$. At the same time, we have the equality $\mathcal{I}\left(\mu^{\prime}\right)=\mathcal{I}(\mu)$; i.e., $\mu^{\prime}$ does not produce new nullsets.

Both of these constructions do not essentially change the metrical structure of $\mu$. One can observe that the metric space associated with a measure $\mu$ and the metric space associated with its extension $\mu^{\prime}$ obtained by using any of the two described constructions have the same topological weight. Therefore, if the original measure $\mu$ is separable (i.e., its metric space is separable), then the extended measure $\mu^{\prime}$ is separable, too. Kakutani and Oxtoby [3] gave a 
construction of a nonseparable extension of the Lebesgue measure $\lambda$ on the real line $\mathbb{R}$. Another construction of this kind was presented by Kodaira and Kakutani [4]. Both of those extensions of $\lambda$ are invariant under the group of all isometries of $\mathbb{R}$.

It should be observed that the extension of $\lambda$ obtained by Kakutani and Oxtoby has character $2^{\mathrm{c}}$ and necessarily yields new nullsets; i.e., there appear nullsets which are not of Lebesgue measure zero. This is so because the method of Kakutani and Oxtoby uses an uncountable independent family of subsets of $\mathbb{R}$ such that the intersection of any countable subfamily of this family is a Lebesgue nonmeasurable set which becomes a nullset with respect to the extended measure (for more details, see [3]).

The extension of $\lambda$ obtained by Kodaira and Kakutani in [4] has character c. By applying their method, one can also obtain new nullsets (see, for instance, Theorem 2 below). In this connection, the following question seems to be interesting: does there exist a nonseparable extension of $\lambda$ whose nullsets are precisely the $\lambda$-nullsets? The question is of interest in view of the following circumstance: in many topics of real analysis, measure theory, and probability, the inner structure of a measure under consideration does not play any role, and only the induced concept "almost everywhere" is essential. The standard example of this type is a well-known theorem of Lebesgue stating that a bounded function $f:[a, b] \rightarrow \mathbb{R}$ is integrable in the Riemann sense if and only if $f$ is continuous almost everywhere on $[a, b]$. The just mentioned Lebesgue theorem does not need the notion of the Lebesgue measure. For its proof, it completely suffices to apply the notion of a nullset in the Lebesgue sense. Numerous other examples of this kind can be pointed out.

Our goal is to demonstrate (under the Continuum Hypothesis) that there exists a nonseparable extension of $\lambda$ which yields no new nullsets. It should be noticed that our argument may be regarded as a certain combination of the method of Kodaira and Kakutani [4] with the method of Luzin by means of which he proved the existence of his set on the real line (see, e.g., [5], Chapter 20).

We begin with some preliminary considerations.

Let $\left(E_{1}, \mathcal{S}_{1}, \mu_{1}\right)$ and $\left(E_{2}, \mathcal{S}_{2}, \mu_{2}\right)$ be two measure spaces, such that $\mu_{1}$ is $\sigma$-finite and $\mu_{2}$ is a probability measure. Let $f: E_{1} \rightarrow E_{2}$ be a mapping, and let $G(f)$ denote the graph of $f$. Suppose that $G(f)$ is $\left(\mu_{1} \times \mu_{2}\right)$-thick in the product set $E_{1} \times E_{2}$; i.e., suppose that

$$
\left(\mu_{1} \times \mu_{2}\right)_{*}\left(\left(E_{1} \times E_{2}\right) \backslash G(f)\right)=0 .
$$

For any set $Z \in \operatorname{dom}\left(\mu_{1} \times \mu_{2}\right)$, let us define

$$
Z^{\prime}=\left\{x \in E_{1}:(x, f(x)) \in Z\right\} .
$$


Further, introduce a class of sets

$$
\mathcal{S}_{1}^{\prime}=\left\{Z^{\prime}: Z \in \operatorname{dom}\left(\mu_{1} \times \mu_{2}\right)\right\},
$$

and define a functional

$$
\mu_{1}^{\prime}\left(Z^{\prime}\right)=\left(\mu_{1} \times \mu_{2}\right)(Z) \quad\left(Z \in \operatorname{dom}\left(\mu_{1} \times \mu_{2}\right)\right) .
$$

Then $\mathcal{S}_{1}^{\prime}$ is a $\sigma$-algebra of subsets of $E_{1}$, and the functional $\mu_{1}^{\prime}$ is a measure on $\mathcal{S}_{1}^{\prime}$ extending $\mu_{1}$ (cf. [4], p. 576). In addition, the mapping $f$ turns out to be measurable with respect to the $\sigma$-algebras $\mathcal{S}_{1}^{\prime}$ and $\mathcal{S}_{2}$; i.e., for any set $Y \in \mathcal{S}_{2}$, we have $f^{-1}(Y) \in \mathcal{S}_{1}^{\prime}$.

The remarks just made are rather simple, but they will be useful for our further constructions.

Lemma 1. If a measure $\mu_{1}$ is nonzero and a measure $\mu_{2}$ is nonseparable, then the measure $\mu_{1}^{\prime}$ is nonseparable, too.

Proof. Take any set $X \in \operatorname{dom}\left(\mu_{1}\right)$ with $0<\mu_{1}(X)<+\infty$. The nonseparability of $\mu_{2}$ implies that, for some $\varepsilon>0$, there exists an uncountable family $\left\{Y_{i}: i \in I\right\}$ of $\mu_{2}$-measurable sets such that

$$
\mu_{2}\left(Y_{i} \triangle Y_{j}\right)>\varepsilon \quad(i \in I, j \in I, i \neq j)
$$

Obviously, we have

$$
\begin{aligned}
& \mu_{1}^{\prime}\left(\left(X \times Y_{i}\right)^{\prime} \triangle\left(X \times Y_{j}\right)^{\prime}\right)=\left(\mu_{1} \times \mu_{2}\right)\left(X \times\left(Y_{i} \triangle Y_{j}\right)\right)= \\
& \mu_{1}(X) \cdot \mu_{2}\left(Y_{i} \triangle Y_{j}\right)>\mu_{1}(X) \cdot \varepsilon \quad(i \in I, j \in I, i \neq j),
\end{aligned}
$$

whence it follows that $\mu_{1}^{\prime}$ is nonseparable.

Let $J$ be a set with $\operatorname{card}(J)=\mathbf{c}$. For any index $j \in J$, denote by $\nu_{j}$ the restriction of the Lebesgue measure $\lambda$ to the Borel $\sigma$-algebra of $[0,1]$. Thus, $\nu_{j}$ is a Borel probability measure on $[0,1]$. Let $\nu$ stand for the product measure $\prod_{j \in J} \nu_{j}$

The following two auxiliary propositions are easy and well known, but, for the sake of completeness, we give their short proofs here.

Lemma 2. The cardinality of dom $(\nu)$ is equal to $\mathbf{c}$, and the topological weight of the metric space associated with $\nu$ is also equal to $\mathbf{c}$. In particular, $\nu$ is a nonseparable measure. 
Proof. Any set $Z \in \operatorname{dom}(\nu)$ can be represented in the form $B \times[0,1]^{J \backslash J_{0}}$, where $J_{0}$ is a countable subset of $J$ (certainly, depending on $Z$ ) and $B$ is a Borel subset of $[0,1]^{J_{0}}$. Taking into account the equality $\mathbf{c}^{\omega}=\mathbf{c}$, this readily implies that $\operatorname{card}(\operatorname{dom}(\nu))=\mathbf{c}$.

Further, for each $j \in J$, let us denote

$$
Z_{j}=[0,1 / 2]_{j} \times[0,1]^{J \backslash\{j\}} .
$$

Then we have

$$
\nu\left(Z_{j} \triangle Z_{k}\right)=1 / 2 \quad(j \in J, k \in J, j \neq k),
$$

whence it follows that the topological weight of the metric space associated with $\nu$ is equal to $\mathbf{c}$ (in other words, $\nu$ has character $\mathbf{c}$ ). This ends the proof of Lemma 2.

Lemma 3. Let $\alpha$ be an infinite cardinal number satisfying the relation $\alpha^{\omega}=\alpha$, and let $\left(E_{1}, \mathcal{S}_{1}\right)$ and $\left(E_{2}, \mathcal{S}_{2}\right)$ be two measurable spaces such that $\operatorname{card}\left(\mathcal{S}_{1}\right) \leq \alpha$ and $\operatorname{card}\left(\mathcal{S}_{2}\right) \leq \alpha$. Then the cardinality of the product $\sigma$-algebra $\mathcal{S}_{1} \otimes \mathcal{S}_{2}$ does not exceed $\alpha$, either.

Proof. The product $\sigma$-algebra $\mathcal{S}_{1} \otimes \mathcal{S}_{2}$ is generated by the family of sets $\mathrm{Z}$ having the form $Z=X \times Y$, where $X \in \mathcal{S}_{1}$ and $Y \in \mathcal{S}_{2}$. The cardinality of this family does not exceed $\alpha \cdot \alpha=\alpha$. Taking into account the equality $\alpha^{\omega}=\alpha$, we come to the required result.

The next lemma plays a key role in our further consideration.

Lemma 4. Assume the Continuum Hypothesis. There exists a mapping

$$
f: \mathbb{R} \rightarrow[0,1]^{J}
$$

satisfying the following relations:

(1) the graph of $f$ is thick with respect to the product measure $\lambda \times \nu$;

(2) for any $(\lambda \times \nu)$-measure zero set $Z$, the set $Z^{\prime}=\{x:(x, f(x)) \in Z\}$ is of $\lambda$-measure zero.

Proof. The required mapping $f$ will be constructed by transfinite recursion.

In what follows, the symbol $\lambda_{0}$ stands for the restriction of the Lebesgue measure to the Borel $\sigma$-algebra of $\mathbb{R}$.

Let $\alpha$ denote the least ordinal number of cardinality $\mathbf{c}$. According to our assumption, $\alpha=\omega_{1}$; i.e., $\operatorname{card}(\xi) \leq \omega$ for each ordinal $\xi<\alpha$.

Let $\preceq$ denote a well-ordering of $\mathbb{R}$ which is isomorphic to $\alpha$. 
Applying Lemmas 2 and 3, we deduce the equality

$$
\operatorname{card}\left(\operatorname{dom}\left(\lambda_{0} \times \nu\right)\right)=\mathbf{c} .
$$

Let $\left\{Z_{\xi}: \xi<\alpha\right\}$ be the family of all those $\left(\lambda_{0} \times \nu\right)$-measurable sets whose measure is strictly positive. We may suppose, without loss of generality, that the range of this family coincides with the range of $\left\{Z_{\xi}: \xi<\alpha, \xi\right.$ is odd $\}$.

Let $\left\{T_{\xi}: \xi<\alpha\right\}$ be an enumeration of all those $\left(\lambda_{0} \times \nu\right)$-measurable sets whose measure is equal to zero.

We are going to define an $\alpha$-sequence $\left\{\left(x_{\xi}, y_{\xi}\right): \xi<\alpha\right\}$ of points of the product space $\mathbb{R} \times[0,1]^{J}$. Suppose that, for an ordinal $\xi<\alpha$, the partial $\xi$-sequence $\left\{\left(x_{\zeta}, y_{\zeta}\right): \zeta<\xi\right\}$ has already been constructed. Consider two cases.

(a). The ordinal $\xi$ is even. In this case, denote by $x$ the $\preceq$-least element of the set $\mathbb{R} \backslash\left\{x_{\zeta}: \zeta<\xi\right\}$ and, for each ordinal $\zeta<\xi$, define

$$
T_{\zeta}(x)=\left\{y:(x, y) \in T_{\zeta}\right\} .
$$

Also, define a set $\Xi$ by the formula

$$
\Xi=\left\{\zeta: \zeta<\xi \& \nu\left(T_{\zeta}(x)\right)=0\right\}
$$

Obviously, we have $\nu\left(\cup\left\{T_{\zeta}(x): \zeta \in \Xi\right\}\right)=0$. Consequently,

$$
[0,1]^{J} \backslash \cup\left\{T_{\zeta}(x): \zeta \in \Xi\right\} \neq \emptyset .
$$

Choose a point $y \in[0,1]^{J} \backslash \cup\left\{T_{\zeta}(x): \zeta \in \Xi\right\}$, and put $\left(x_{\xi}, y_{\xi}\right)=(x, y)$.

(b). The ordinal $\xi$ is odd. For each ordinal $\zeta<\xi$, define

$$
T_{\zeta}^{0}=\left\{x \in \mathbb{R}: \nu\left(T_{\zeta}(x)\right)>0\right\} .
$$

Since $\left(\lambda_{0} \times \nu\right)\left(T_{\zeta}\right)=0$, we have $\lambda\left(T_{\zeta}^{0}\right)=0$. Consider the set $Z_{\xi}$. Taking into account that $\left(\lambda_{0} \times \nu\right)\left(Z_{\xi}\right)>0$ and applying the Fubini theorem, we can find a point $x \in \mathbb{R} \backslash \cup\left\{T_{\zeta}^{0}: \zeta<\xi\right\}$ satisfying the relation $\nu\left(Z_{\xi}(x)\right)>0$. Choose a point $y \in Z_{\xi}(x) \backslash \cup\left\{T_{\zeta}(x): \zeta<\xi\right\}$, and put $(x, y)=\left(x_{\xi}, y_{\xi}\right)$.

Thus, in both cases (a) and (b), we have defined $\left(x_{\xi}, y_{\xi}\right)$. Proceeding in this manner, we are able to construct the $\alpha$-sequence $\left\{\left(x_{\xi}, y_{\xi}\right): \xi<\alpha\right\}$ of points of $\mathbb{R} \times[0,1]^{J}$. Now, let us put

$$
f\left(x_{\xi}\right)=y_{\xi} \quad(\xi<\alpha) .
$$

We assert that the function $f$ is the required one. Indeed, the equality

$$
\mathbb{R}=\left\{x_{\xi}: \xi<\alpha\right\}
$$


holds true (because the well-ordering $\preceq$ is isomorphic to $\alpha$ ). Therefore, the domain of $f$ coincides with $\mathbb{R}$. The thickness of $G(f)$ is a straightforward consequence of the relations

$$
\left(x_{\xi}, y_{\xi}\right) \in Z_{\xi} \quad(\xi<\alpha, \xi \text { is odd }) .
$$

Finally, let us show that the inclusion

$$
\left\{x:(x, f(x)) \in T_{\xi}\right\} \subset T_{\xi}^{0} \cup\left\{x_{\zeta}: \zeta \leq \xi\right\}
$$

is valid for each ordinal $\xi<\alpha$. Take any $(x, f(x)) \in T_{\xi}$. According to our construction, $(x, f(x))=\left(x_{\eta}, y_{\eta}\right)$ for some $\eta<\alpha$. Consequently,

$$
\left(x_{\eta}, y_{\eta}\right) \in T_{\xi}, \quad y_{\eta} \in T_{\xi}\left(x_{\eta}\right) .
$$

If $\eta \leq \xi$, then there is nothing to prove. Suppose now that $\xi<\eta$ and consider two cases.

(i). $\nu\left(T_{\xi}\left(x_{\eta}\right)\right)=0$. If $\eta$ is even, then our construction yields $y_{\eta} \notin T_{\xi}\left(x_{\eta}\right)$. If $\eta$ is odd, then our construction also yields $y_{\eta} \notin T_{\xi}\left(x_{\eta}\right)$. Therefore, this case is impossible for $\xi<\eta$.

(ii). $\nu\left(T_{\xi}\left(x_{\eta}\right)\right)>0$. This relation immediately implies $x_{\eta} \in T_{\xi}^{0}$, which yields the desired result.

The proof of Lemma 4 is thus completed.

Theorem 1. Under the Continuum Hypothesis, there exists a nonseparable measure $\lambda^{\prime}$ on the real line $\mathbb{R}$ extending $\lambda$ such that $\mathcal{I}\left(\lambda^{\prime}\right)=\mathcal{I}(\lambda)$.

Proof. In view of Lemma 4, there is a mapping

$$
f: \mathbb{R} \rightarrow[0,1]^{J}
$$

whose graph is thick in the product space $\mathbb{R} \times[0,1]^{J}$. For any $Z \in \operatorname{dom}\left(\lambda_{0} \times \nu\right)$, we put

$$
Z^{\prime}=\{x:(x, f(x)) \in Z\}, \quad \lambda^{\prime}\left(Z^{\prime}\right)=\left(\lambda_{0} \times \nu\right)(Z) .
$$

As said earlier, the functional $\lambda^{\prime}$ is well defined and is a measure extending $\lambda_{0}$. Obviously, the completion of $\lambda^{\prime}$ extends the Lebesgue measure on $\mathbb{R}$. We preserve the same notation for the completion of $\lambda^{\prime}$. By virtue of Lemmas 1 and $2, \lambda^{\prime}$ is a nonseparable measure. More precisely, we can assert that the character of $\lambda^{\prime}$ is equal to $\mathbf{c}$. This ends the proof of the theorem.

Remark 1. Theorem 1 was established by assuming the Continuum Hypothesis. One of the referees kindly informed the author that the statement of this theorem remains valid under Martin's Axiom and even under a weaker assumption on cardinal invariants associated with $\mathcal{I}(\nu)$ and $\mathcal{I}(\lambda)$. The same 
referee also mentioned that the existence of a nonseparable extension of $\lambda$ without new nullsets cannot be proved within ZFC theory. In this connection, it should be underlined that there is (within ZFC) a proper separable extension of $\lambda$ whose nullsets are identical with the $\lambda$-nullsets (cf. Example 2).

Remark 2. As pointed out at the end of the proof of Theorem 1, the character of $\lambda^{\prime}$ is equal to c. We do not know whether it is possible to show (at least, in some models of set theory) the existence of an extension of $\lambda$ whose character is strictly greater than $\mathbf{c}$ and whose nullsets coincide with the nullsets in the Lebesgue sense.

Let $(E, \mathcal{S}, \mu)$ be a probability measure space. The argument presented before Lemma 1 shows that if a mapping $g: \mathbb{R} \rightarrow E$ is given whose graph is thick in $\mathbb{R} \times E$, then this mapping produces the measure $\lambda_{g}$ which is an extension of the Lebesgue measure $\lambda$.

Theorem 2. Suppose that $\operatorname{card}(\mathcal{S}) \leq \mathbf{c}$. Then there exists a mapping $h: \mathbb{R} \rightarrow$ $E$ satisfying the following conditions:

(1) the graph of $h$ is thick in the product space $\mathbb{R} \times E$;

(2) some set of $\lambda_{h}$-measure zero is thick in $\mathbb{R}$ with respect to $\lambda$; in particular, $\mathcal{I}\left(\lambda_{h}\right) \neq \mathcal{I}(\lambda)$.

Proof. Let $B$ be a Bernstein subset of $\mathbb{R}$. We recall that, according to the definition of Bernstein sets, both of the sets $B$ and $\mathbb{R} \backslash B$ are totally imperfect in $\mathbb{R}$, which readily implies the equalities

$$
\begin{gathered}
\operatorname{card}(B)=\operatorname{card}(\mathbb{R} \backslash B)=\mathbf{c}, \\
\lambda_{*}(B)=\lambda_{*}(\mathbb{R} \backslash B)=0 .
\end{gathered}
$$

In particular, $B$ and $\mathbb{R} \backslash B$ are not measurable in the Lebesgue sense. Choose any point $y \in E$ and take the constant mapping $h_{0}: B \rightarrow\{y\}$. By using the method of transfinite recursion, a mapping

$$
h_{1}: \mathbb{R} \backslash B \rightarrow E \backslash\{y\}
$$

can easily be constructed such that the graph $G\left(h_{1}\right)$ is thick in the product space $\mathbb{R} \times E$ (cf. the proof of Theorem 1). Let $h$ stand for the common extension of $h_{0}$ and $h_{1}$. Clearly, the graph of $h$ is also thick in $\mathbb{R} \times E$. Consider the set $\mathbb{R} \times\{y\}$. Since the measure $\lambda$ is $\sigma$-finite and $\mu(\{y\})=0$, we get

$$
(\lambda \times \mu)(\mathbb{R} \times\{y\})=0 .
$$

Consequently, we must have

$$
\lambda_{h}(\{x:(x, h(x)) \in \mathbb{R} \times\{y\}\})=0 .
$$


Therefore, $\{x:(x, h(x)) \in \mathbb{R} \times\{y\}\} \in \mathcal{I}\left(\lambda_{h}\right)$. But it is easy to see the validity of the relation

$$
B=\{x:(x, h(x)) \in \mathbb{R} \times\{y\}\},
$$

whence it follows that the set $\{x:(x, h(x)) \in \mathbb{R} \times\{y\}\}$ is thick with respect to $\lambda$. Moreover, the above-mentioned set being a Bernstein subset of $\mathbb{R}$ is thick with respect to any $\sigma$-finite continuous Borel measure on $\mathbb{R}$.

This completes the proof of Theorem 2 .

The author is grateful to the referees for their remarks, suggestions, and improvements.

\section{References}

[1] A. Ascherl, J. Lehn, Two principles for extending probability measures, Manuscripta Math., 21 (1977), 43-50.

[2] D. Bierlein, Über die Fortsetzung von Wahrscheinlichkeitsfeldern, Z. Wahrsch. Verw. Gebiete, 1 (1962), 28-46.

[3] S. Kakutani, J. C. Oxtoby, Construction of a nonseparable invariant extension of the Lebesgue measure space, Ann. of Math., 52 (1950), 580-590.

[4] S. Kodaira, S. Kakutani, A nonseparable translation-invariant extension of the Lebesgue measure space, Ann. of Math., 52 (1950), 574-579.

[5] J. C. Oxtoby, Measure and Category, Springer-Verlag, Berlin, 1971.

[6] W. F. Pfeffer, K. Prikry, Small spaces, Proc. London Math. Soc., 58 (1989), 417-438.

[7] E. Szpilrajn (E. Marczewski), Sur l'extension de la mesure lebesguienne, Fund. Math., 25 (1935), 551-558.

[8] S. Ulam, Zur Masstheorie in der allgemeinen Mengenlehre, Fund. Math., 16 (1930), 140-150.

[9] P. Zakrzewski, On a construction of universally small sets, Real Anal. Exchange, 28(1) (2002/03), 221-226. 